\section{A Precise Determination of the Fine Structure Constant}

About a year ago, R. T. Birge ${ }^{1}$ suggested the possibility of an experimental determination of the fine structure constant based on measurements of the x-ray spin doublet splitting ( $L_{\mathrm{II}}-L_{\mathrm{III}}$ levels). A direct determination of this quantity is of interest because of the discrepancies existing in the values of the atomic constants, and because of controversial ideas as to the value of this constant itself. Birge assembled data on the doublet splitting as obtained in several different $x$-ray lines, and in elements varying in atomic number from $Z=29$ to 92 . He showed that these data formed a body of material of sufficient accuracy and consistency for such a determination.

We have recently completed calculations giving the theoretical behavior of the spin doublet splitting for elements of high atomic number. The Sommerfeld formula for the energy difference between the $2 P_{1 / 2}$ and $2 P_{3 / 2}$ levels (in units of $m c^{2}$ ),

$$
S(\alpha Z)=\frac{1}{2}\left\{4-(\alpha Z)^{2}\right\}^{\frac{1}{3}}-\frac{1}{2}\left\{2+2\left[1-(\alpha Z)^{2}\right]^{\frac{1}{2}}\right\}^{\frac{1}{2}}
$$

gives the splitting due to the Coulomb field of the nucleus alone. The effect of the electrons on the splitting is of order $1 / Z$ compared to that of the nuclear charge because only those few electrons lying close to the nucleus contribute essentially. Thus we can calculate the effect of the electrons by perturbation theory, where $1 / Z$ is the parameter of expansion. We have carried out this calculation, using the Dirac wave functions in a Coulomb field of charge $Z e$ for our zero-order wave functions. Complete interactions of electrons of the $K$ and $L$, and direct interactions of the $M$ shell on the $2 P_{1 / 2}$ and $2 P_{3 / 2}$ states have been carried out in first order, with the use of the Breit Hamiltonian.

We thus get the interaction energy of the electrons as a function of $\alpha Z$. The first-order term can be obtained to an arbitrary accuracy, both in magnitude and in its functional dependence on $\alpha Z$. In the higher order terms in $1 / Z$, the lowest term in $\alpha Z$ could in principle be determined by higher order perturbation calculation. Further terms in $\alpha Z$ cannot be so determined because of fundamental limitations of present quantum-electrodynamic theory. But these terms are of order $\alpha^{2}$ times the splitting and are negligible. Actually, we do not calculate even the coefficient of the leading terms in $\alpha Z$ of the second and higher order perturbation terms, but consider it sufficient to introduce arbitrary coefficients, which will be determined by fitting the experimental data.

Assuming that the Rydberg constant is given correctly by $R=2 \pi^{2} m e^{4} / h^{3}$, we represent the experimental data in the form

$$
\frac{\Delta \nu}{R Z^{4}}=\frac{2}{\alpha^{2} Z^{4}} S(\alpha Z)-\frac{2 g \alpha^{2} f(\alpha Z)}{Z}+\frac{B \alpha^{2}}{Z^{2}} .
$$

$f(\alpha Z)$ is the first-order term we have calculated. Because we have neglected interactions of electrons beyond the $M$ shell, we have introduced the constant $g$, whose value is nearly unity. We estimate that $g$ may differ from unity by at most one percent. Taking $g$ as unity, we have made a least-squares solution based on Eq. (2), using data from $Z=60$ to 92 . We obtain a value of $1 / \alpha=136.95$, with a. statistical probable error of 0.019 . An error of one percent in $g$ would produce an error of 0.068 in $1 / \alpha$. To obtain an over-all limit of error, we take three times our statistical error and add to it the possible error arising from the uncertainty in $g$. This gives us $1 / \alpha=h c / 2 \pi e^{2}=136.95$, which we believe correct to within 0.13 .

We are indebted to Professor J. R. Oppenheimer for pointing out to us this method of calculating the splitting, and for continual encouragement.

$$
\begin{gathered}
\text { Department of Physics, } \\
\text { University of California, } \\
\text { Berkeley, California, } \\
\text { September 16, } 1940 .
\end{gathered}
$$

1 R. T. Birge, Phys. Rev. 55, 1119 (1939).
R. F. CHRISTY
J. M. KeLLER

\section{The Values of $e, e / m, h / e$ and $\alpha$}

There has existed for several years a fundamental discrepancy between the directly measured values of $e, t / m$ and $h / e$, namely, that the "best" numerical values of these three quantities do not satisfy the Bohr formula for the Rydberg constant. The writer emphasized this point some time ago, ${ }^{1}$ and the situation has since been frequently discussed, particularly by DuMond. Unfortunately there has not been available, thus far, any fourth quantity whose value appeared to be known with sufficient reliability to throw any real light on the discrepancy.

A year ago I showed ${ }^{2}$ that the then available data on the $L_{\text {II }} L_{\text {III }}$ interval, as determined from x-ray wave-lengths, were sufficiently extensive and precise to give a reliable value of the fine structure constant. It was, however, immediately pointed out to me by J. R. Oppenheimer that a really trustworthy value could be obtained only from a detailed study of the matter, on the basis of modern quantum mechanics. Such a study has now been made, under his direction, by J. M. Keller and R. F. Christy, and is reported in the preceding letter. The result is $1 / \alpha=136.95$, with a probable error that I take to be \pm 0.05 . This probable error is about one-tenth that of the best previous determination. The new value of $1 / \alpha$ thus constitutes the desired fourth quantity.

In August, 1939, I prepared a mimeographed list of the general constants, and submitted it to many persons for suggestions and criticism. In preparing the list I used the latest values of all auxiliary constants, and in particular I assumed that the directly observed values of $e$ and $e / m$ were correct, and that the Rydberg constant formula was valid. All recent theoretical work seems to justify this last assumption. The correlated conclusion is then that the seemingly most reliable measurements of $h / e$ (from the Duane-Hunt limit of the continuous $x$-ray spectrum) give a result much too low. The relevant adopted values on my 1939 list are $e=(4.8022 \pm 0.0010) \times 10^{-10}$ e.s.u., $e / m$ $=(1.7591 \pm 0.0005) \times 10^{7} \quad$ e.m.u., $\quad R_{\infty}=109,737.45 \pm 0.06$ $\mathrm{cm}^{-1}$. On the basis of subsequent work by Miller and DuMond, ${ }^{3}$ I deem it best to increase the probable error of $e$ to \pm 0.002 , but for the present I shall not modify the actual value of $e$. One then calculates, from the Rydberg constant formula, $h / e=(1.37929 \pm 0.00040) \times 10^{-17}$, as contrasted with DuMond and Bollman's directly observed ${ }^{4}$ $1.3765 \pm 0.0003$. This is the fundamental discrepancy. 